INVESTIGACIÓN

Recibido: 12/03/2021 --- Aceptado: 15/04/2021 --- Publicado: 24/04/2021

\title{
REVISIÓN TEÓRICO-CIENTÍFICA DEL MARCO CONCEPTUAL DE LA EMOCIÓN Y EL SENTIMIENTO Y SU APLICACIÓN AL NEUROMARKETING
}

\section{Theoretical-scientific review of the conceptual framework of emotion and sentiment and its application to neuromarketing}

Marian Núñez- Cansado. Universidad de Valladolid. España. marian@hmca.uva.es

Aurora López López. Universidad de Valladolid. España. mariaaurora.lopez.lopez@uva.es.

Cristina Vela Delfa. Universidad de Valladolid. Madrid. vela@fyl.uva.es

Como citar el artículo:

Núñez- Cansado, M., López López, A. y Vela Delfa, C. (2021). Revisión teóricocientifícia del marco conceptual de la emoción y el sentimiento y su aplicación al neuromarketing.Vivat Academia. Revista de Comunicación, 154, 381-407. http:/ / doi.org/10.15178/va.2021.154.e1357

\section{RESUMEN}

En primera instancia, este artículo aborda una revisión bibliográfica diacrónica de las principales aportaciones teóricas relativas a la delimitación del marco conceptual de la emoción y el sentimiento, para plantear, finalmente, una reflexión acerca de su aplicación en el ámbito de la investigación en neuromarketing. En los últimos años, los programas de investigación científica han optado, en mayor medida, por desmarcarse del que ha sido durante mucho tiempo el paradigma dominante en las teorías de la emoción -que unificaba el tratamiento de las nociones de emoción y sentimiento-, para inclinarse por definiciones capaces de significar las diferencias existentes entre ambos conceptos, desde una óptica multifactorial y diferencial. Por su parte, el análisis de la literatura científica sobre neuromarketing nos revela que, en la práctica, ambos términos se confunden. Se incurre, de este modo, en un neurocentrismo que ignora el papel de los sentimientos en la toma de decisión. Ciertamente, una gran mayoría de estudios, procedentes de la praxis en este campo, centran sus resultados en aspectos meramente fisiológicos-biológicos, y dejan a un lado datos relevantes relativos a los procesos cognitivos asociados a aspectos culturales, sociales y propios de los estilos cognitivos del sujeto; circunstancia que 
Núñez- Cansado, M., López López, A. y Vela Delfa, C.

Revisión teórico-cientifícia del marco conceptual de la emoción y el sentimiento y su aplicación al neuromarketing

puede restar validez al constructo teórico y limitar bien la capacidad predictiva o la validez concurrente en el diseño de estas investigaciones. Por todo esto, con la revisión bibliográfica propuesta se aportará luz sobre la problemática científica existente en torno al manejo de las nociones de emoción y sentimiento, lo que contribuirá, a su vez, a la adopción de una perspectiva más crítica y acertada acerca de cuáles deberían ser realmente sus usos y aplicaciones en el ámbito de la investigación en general, y del neuromarketing en particular. En este sentido, es importante superar algunas de las actuales limitaciones concernientes a las metodologías mixtas e integradas con las que se mide y observa la actividad cerebral, y para ello las aclaraciones teórico-científicas que resultan de este estudio serán claves y dilucidadoras.

PALABRAS CLAVE: Neuromarketing- Psicología del consumidor- Emoción, Sentimiento- Minería de sentimientos- Marketing emocional- Marketing sentimental.

\section{ABSTRACT}

In this article we try to address a diachronic bibliographical revision of the main theoretical contributions regarding the conceptual frame of emotion and feeling and its use in neuromarketing investigation. In the last years, scientific investigation programs are more prone to break the predominant paradigm in emotion theory, that identifies emotion and feeling, leaning towards definitions more accurate to the distinction of both concepts, proposing a differential and multifaceted perspective. Nonetheless, the analysis of specific literature over neuromarketing points that, in practice, both terms are confused. Consequently, we fall into a neurocentrism that ignores the role that feelings play in decision making. Most studies aim their results in merely physiologic and biological aspects, leaving behind relevant aspects that are the result of cognitive processes largely associated to cultural, social, and cognitive aspects of the subject. This circumstance may take away validity from the theoretical construct, as well as limit its predictive capacity or lessen its actual validity in the design of its investigations. In the current scientifical context, and regarding the bibliographical revision performed through this investigation, we consider the definition of a clear and unified conceptual frame to be crucial, so much as to avoid theoretical misunderstandings as to improve the design of the investigations that have been applied. We consider the investigation in neuromarketing entails a higher scientific accuracy, which implies the realization of more precise and coherent theorical interpretations of an updated conceptual frame. Only this way we will be able to overcome current limitations inherent to the application of mixed and integrated methodologies with which we measure and observe brain activity in the neuromarketing field.

KEYWORDS: Neuromarketing-Consumer psychology- Emotion- Sentiment, Sentiment mining- Emotional marketing, Sentimental marketing. 
Núñez- Cansado, M., López López, A. y Vela Delfa, C.

Revisión teórico-cientifícia del marco conceptual de la emoción y el sentimiento y su aplicación al neuromarketing

\section{INTRODUCCIÓN}

El recorrido por las teorías básicas de la emoción y las aportaciones al respecto desde el ámbito del marketing y el neuromarketing muestran un patrón común en el modo en el cual los conceptos de "emoción" y "sentimiento" son tratados, casi siempre como términos sinónimos. El estudio realizado por el equipo del Dr. Gaur (2014) sobre la revisión de más de 340 artículos de investigación en el ámbito del marketing afirma que términos como "afecto", "sentimientos", "estados de ánimo" y "emociones" son usados indistintamente con frecuencia. De acuerdo con Bagozzi et al. (1999) en su estudio sobre el papel de las emociones en el marketing, esta confusión conlleva la producción de textos científicos sobre las emociones con hallazgos poco concluyentes, cargados de afirmaciones reduccionistas que no reflejan el verdadero avance científico que las neurociencias están experimentando en los últimos años. A nuestro modo de ver, esta circunstancia no es solo una cuestión terminológica, sino que pone de manifiesto la complejidad del estudio de las emociones y su aplicación al estudio del consumidor.

Es posible que el origen de esta confusión parta de un problema metalingüístico, al emplear vocablos del lenguaje ordinario en la creación de taxonomías para las emociones. Muchos investigadores creen que esta es una buena forma de acercar la ciencia al público, pero, como defiende Scarantino (2018), esta circunstancia no es la mejor para reforzar la cientificidad de la disciplina y no puede justificar que las emociones sean definidas como sentimientos en la investigación científica. El resultado de esta actitud es la falta de precisión en la definición de ambos conceptos: sentimiento y emoción, lo que provoca malentendidos científicos acerca de su manejo, tal y como demuestra este trabajo.

\section{OBJETIVOS}

El principal objetivo de este artículo es realizar una revisión bibliográfica sobre el modo en que los conceptos de emoción y sentimiento han sido y son abordados en la literatura científica, a fin de delimitarlos conceptualmente de manera adecuada. Se parte de considerar que, hasta ahora, tanto desde la teoría como desde la praxis, ambos han sido tratados erróneamente como términos sinónimos. Por ello, a través de un recorrido histórico desde la antigüedad hasta las teorías actuales sobre su evolución conceptual, se contemplan los avances en la materia y las repercusiones que conllevan en la práctica del neuromarketing.

\section{METODOLOGÍA}

En este trabajo se lleva a cabo una revisión bibliográfica, o estado del arte, sobre los dos conceptos objeto de estudio, a saber: emoción y sentimiento, para determinar cuál ha sido la evolución conceptual de su marco teórico-científico y las distintas implicaciones de todo ello en las acciones de neuromarketing. Así, en una primera fase se procede a una búsqueda exhaustiva de información sobre el tratamiento que han recibido ambos conceptos desde diferentes corrientes y perspectivas científicas.

Vivat Academia. Revista de Comunicación. 2021, nº 154, 381-407 
Núñez- Cansado, M., López López, A. y Vela Delfa, C.

Revisión teórico-cientifícia del marco conceptual de la emoción y el sentimiento y su

aplicación al neuromarketing

En una segunda fase se procede a una exploración de la literatura científica en el ámbito del neuromarketing, relativa a la métrica de la emoción y de los sentimientos. La tercera fase se orienta al análisis de la información obtenida, en las dos fases anteriores, para poner de manifiesto los elementos de discusión que subyacen de la descripción de ambos conceptos según diferentes modelos teóricos. Asimismo, se abordarán los usos del neuromarketing en torno a ambos términos en el planteamiento de acciones concretas y la consecución de determinados objetivos.

Para terminar, se concluye con una serie de inferencias, que permiten valorar diferentes escenarios futuros sobre los que considerar la continuidad de la disciplina del neuromarketing.

\section{REVISIÓN BIBLIOGRÁFICA}

\subsection{Un breve recorrido por las teorías de la emoción y el sentimiento}

Desde los inicios de la historia de la filosofía occidental, la emoción, puesta en confrontación con el concepto de razón, ha sido un elemento clave en la investigación de múltiples disciplinas. Emoción y cognición han sido enfrentadas en un dualismo antropológico que otorgaba a la razón el rol responsable de comportamientos intelectuales, mientras que la emoción quedaba relegada a rutas y alternativas erróneas: "la educación emocional no es un invento tan moderno como parece. Sócrates, Platón, Aristóteles, Epicuro, Epicteto o Séneca fueron educadores de los sentimientos" (Mahamud, 2018).

Desde la filosofía clásica occidental y hasta mediados del siglo XIX, los términos usados para conceptualizar la emoción eran "pasión", "sentimientos" o "afección". De hecho, no será hasta décadas recientes cuando la palabra emoción comienza a popularizarse en la bibliografía (Dixon, 2003).

Platón describe la pasión y la razón en la alegoría del carro alado. A través de esta metáfora, presente en el diálogo de Fedro (Sección 246 a-254e) (Magnone, 2012), trata de explicar la naturaleza del alma humana, que es descrita atendiendo a tres dimensiones básicas: razón, apetito (que empuja hacia los goces de los malos placeres) y espíritu. Cada una de estas dimensiones platónicas se corresponderían con lo que actualmente se denomina cognición, motivación y emoción, respectivamente.

Será Aristóteles quien presente una primera visión bastante acertada sobre la actual noción de emoción. No en vano, Aristóteles es considerado el padre de la psicología cognitiva (Arnold, 1960). Para Aristóteles las dos dimensiones del alma forman un único elemento, las afecciones del alma se reflejan en las del cuerpo, pues tanto en el miedo, en la compasión o en la osadía, el cuerpo está implicado y es parte afectada por sus consecuencias (Trueba, 2009). Desde su punto de vista, las emociones son elementos racionales, como creencias y expectativas. Esta novedosa 
Núñez- Cansado, M., López López, A. y Vela Delfa, C.

Revisión teórico-cientifícia del marco conceptual de la emoción y el sentimiento y su aplicación al neuromarketing

perspectiva supone la inclusión de los elementos cognitivos y valorativos en el concepto de emoción que, años más tarde, heredarán los psicólogos cognitivistas. Según Aristóteles las emociones son parte esencial de nuestra vida, lo que le lleva a considerar, por ejemplo, la insensibilidad hacia el placer o el dolor como un vicio o una deficiencia.

Esta visión presentada por el estagirita no será retomada hasta el siglo XX, ya que durante gran parte de la edad media y la edad moderna seguirá imperando la concepción funcionalista. En las definiciones sigue presente el dualismo e incluso el estigma de culpabilidad de las emociones con relación a las decisiones erróneas y los principales problemas humanos, tal y cómo defendían los estoicos. En cierta manera, la Edad Media y la llegada del cristianismo relegó a la emoción al terreno de lo pecaminoso:

La Edad Media fue una etapa en la que se tenía una idea negativa de la existencia, pesimista, un "valle de lágrimas" en el que las emociones positivas no tenían cabida. La mayoría de las teorías medievales ligaban las emociones a las pasiones, apetitos y deseos, considerándolas como algo que se debía controlar (Bisquerra, 2000, p. 31).

Es más, aún transcurrida la Edad Media, algunos filósofos siguen otorgando connotaciones negativas a la emoción y le restan poder frente a la razón. Hobbes reconoce cuatro facultades fundamentales en el ser humano: la fuerza física, la experiencia, la razón y la emoción (identificada con la pasión). Este filósofo considera que las pasiones son el principio del movimiento del ser humano y que el pensamiento está a su servicio, provocando decisiones nubladas no racionales (Vargas \& Espinoza, 2008), que se dirigen hacia la búsqueda del placer y la evitación del dolor.

Para Descartes, quién ubica el alma en la glándula pineal, en el mismo lugar que localiza la emoción, sigue existiendo un dualismo claro: mente-cuerpo y razónemoción. En su modelo, la razón debía estar distanciada de la emoción y podía llevarnos a tomar decisiones totalmente erróneas. El filósofo considera que existen seis emociones simples y primitivas: el asombro, el amor, el odio, el deseo, la alegría y la tristeza y que todas las demás están compuestas de estas seis o son derivados de ellas.

Será un filósofo coetáneo de Descartes, Spinoza, quién rompa con esta dualidad y siga los principios más básicos de Aristóteles, considerando la emoción como elemento tanto del alma como del cuerpo, e incluso planteando que ambas llegan a la fusión: "El alma y el cuerpo es una y la misma cosa, que es concebida ora bajo el atributo del pensamiento, ora bajo el de la extensión. De donde resulta que el orden o concatenación de las cosas es uno solo" (González, 2017. S.p.).

Spinoza no acepta las visiones planteadas por Descartes, o por autores de la Edad Media como San Agustín o Santo Tomás. No solo rechaza el dualismo sino que, además, deja de lado el concepto de emoción como causa de imperfección o error.

Vivat Academia. Revista de Comunicación. 2021, nº 154, 381-407 
Núñez- Cansado, M., López López, A. y Vela Delfa, C.

Revisión teórico-cientifícia del marco conceptual de la emoción y el sentimiento y su aplicación al neuromarketing

Propone una clasificación de las emociones basada en tres tipologías diferentes: las emociones de fondo, emociones que no son especialmente fáciles de ver, y que consisten más en reacciones reguladoras del organismo que se pueden detectar, como mal humor o entusiasmo; las emociones primarias o básicas: serían aquellas que comparten la mayor parte de las culturas y son fáciles de identificar, entre ellas, el miedo, la ira, el asco, la sorpresa, la tristeza, la felicidad, etc.; y finalmente, las emociones sociales, que serían mezcla entre las dos categorías anteriores e incluirían algunas como: la simpatía, la turbación, la vergüenza, la culpabilidad, el orgullo, los celos, la envidia, la gratitud, la admiración, la indignación y el desdén (González, 2017).

Un siglo más tarde, el filósofo David Hume introduce una nueva perspectiva del concepto de la emoción, que describe como una sensación susceptible de medirse al igual que cualquier otro fenómeno físico. En la línea de Aristóteles, concede un valor crucial a los aspectos cognitivos, incidiendo en el peso que adquieren las creencias del individuo sobre el estímulo que origina la emoción.

Pero las emociones no solo son analizadas desde la filosofía, sino que han sido objeto de estudio central para disciplinas como la biología, la psicología, la sociología o la antropología. Uno de los pioneros en el estudio de la emoción desde el campo de la biología fue Charles Darwin. Su objeto de estudio se centró en el análisis de las emociones humanas desde su función adaptativa y hereditaria. Describió como las emociones estaban asociadas a las expresiones faciales y eran expresadas de distintas maneras a través de los gestos comunes en hombres e incluso en animales. Esta descripción permitió identificar las emociones básicas que componen el repertorio de las expresiones. Darwin fue además el primero en investigar sobre la universalidad de las emociones:

(...) tanto los animales jóvenes como los viejos expresan igual sus sentimientos, que no es difícil advertir cuán sorprendente es que un perrito pequeño pueda mover la cola cuando está contento, bajar las orejas y descubrir los colmillos cuando quiere mostrarse salvaje, exactamente igual que un perro adulto; o que un gato de corta edad arquee su pequeño lomo y erice el pelo cuando se asusta o se irrita, como un gato mayor. Muchas veces, cuando dirigimos la atención hacia los gestos menos comunes en nosotros mismos, los cuales acostumbramos a ver como artificiales o convencionales encogernos de hombros como signo de impotencia, o elevar los brazos con las manos abiertas y los dedos extendidos en señal de admiración- quizá sintamos demasiada sorpresa ante el descubrimiento de que estas manifestaciones son innatas (Citado en Souza Baelar, 2011, p. 8).

La discusión que separaba razón y emoción comienza así a desparecer y dar el relevo a otras perspectivas como la evolucionista, que abren paso a nuevos marcos conceptuales en el campo de la genética y la psicología social. Tanto la genética como la psicología social pueden considerarse precursoras también de corrientes como la Teoría de las Emociones de Paul Ekman, o la hipótesis del Feedback facial (Díaz Ortíz, 2010), que correlacionan las emociones y los movimientos musculares, en 
Núñez- Cansado, M., López López, A. y Vela Delfa, C.

Revisión teórico-cientifícia del marco conceptual de la emoción y el sentimiento y su aplicación al neuromarketing

especial de la cara, encargados de expresar la emoción. Esta activación muscular puede generar retroalimentación sensorial hacia el cerebro, lo que nos permite sentir la emoción.

Una vez parece superado el dualismo cuerpo-mente y reconocido el nuevo enfoque de estudio aportado por las corrientes naturalistas y conductuales, encabezadas por autores como Darwin, comienzan a identificarse las emociones como parte esencial de nuestra expresión corporal y de todo lo que en el cuerpo ocurre.

Empieza aquí una nueva era, en la que los enfoques psicofisiológicos y neurológicos ocupan un lugar principal en la investigación de las emociones. El estudio de la emoción desde el enfoque psicofisiológico toma como referente al investigador James, quien en el año 1884 señaló que: "los cambios corporales siguen directamente a la percepción del hecho desencadenante (...) nuestra sensación de esos cambios según se van produciendo es la emoción" (James, 1985, p. 60). Desecha así toda posibilidad de contemplar las emociones desde el plano de la cognición. Para James, el sentimiento era más bien una consecuencia que un antecedente de cambios fisiológicos periféricos elicitados por estímulos. Las únicas emociones que podrían ser consideradas como tal eran aquellas que provocaban una expresión corporal distinta (James, 1985).

La discusión queda en estos momentos centrada en la localización de las estructuras que actúan en el proceso de la emoción, a partir de un matiz puramente fisiológico y biológico. Según la teoría elaborada por James y Lange, autor que cita a Spinoza como precursor de su teoría (Vigotsky, 2004), las emociones son el resultado de reacciones fisiológicas a estímulos. En su teoría, ambos autores defienden que la corteza cerebral recibe e interpreta los estímulos ambientales y estos, a su vez, provocan emociones que generan cambios corporales. Es la reacción fisiológica que el sujeto presenta ante el estímulo la que provoca la emoción. Se abre de este modo un debate entre los teóricos, que no se cerrará hasta principio del siglo XXI, y que ya había comenzado Watson: Somos felices porque reímos, o reímos porque somos felices. Así, en respuesta a las afirmaciones de James, Cannon y Bard concluyen en sus investigaciones que se pueden experimentar reacciones fisiológicas al sentir una emoción. Este debate hoy en día ha quedado resuelto y se consideran que ambas posturas son correctas (Fernández et al., 2007).

Al igual que Cannon y Bard, el neurólogo James Papez defiende, en su Teoría del Circuito, que la información sensorial es recibida por el tálamo y dirigida hacia la corteza cerebral y el hipotálamo, responsable de producir las respuestas emocionales. Para Papez (1995) la emoción se define a partir de dos canales básicos: el canal del pensamiento, vía por la que se transmiten los datos sensoriales recibidos a través de los sentidos, donde viajarán del tálamo hacia zonas del neurocórtex (estructura responsable de recepcionar las sensaciones y convertirlas en percepciones, pensamientos o recuerdos). La segunda vía, se define como el canal de los sentimientos, ruta a través de la cual se genera la experiencia subjetiva. Una nueva 
Núñez- Cansado, M., López López, A. y Vela Delfa, C.

Revisión teórico-cientifícia del marco conceptual de la emoción y el sentimiento y su aplicación al neuromarketing

aportación que será vital para comprender el papel crucial de los sentimientos en la toma de decisiones.

A raíz de estas investigaciones, el médico norteamericano Paul MacLean desarrollará su teoría evolutiva sobre el Cerebro Triuno, una de las teorías más utilizadas en el ámbito del neuromarketing. Su propuesta parte de la consideración del cerebro humano a partir de la descripción de tres estructuras: el sistema reptiliano, el sistema límbico y la neocorteza. Otorga al sistema límbico el poder sobre el control de las emociones, incluyendo las estructuras cerebrales descritas en el circuito de Papez. Describe esta estructura a través del desarrollo filogenético de las neuronas, como proceso adaptativo clave en nuestra supervivencia. A pesar de ser una de las teorías de referencia en el ámbito del neuromarketing, sus postulados han quedado relegados a meras hipótesis en relación con la identificación del sistema límbico como estructura responsable de la integración de la información procedente de distintos órganos.

Para LeDoux (1996) el sistema límbico es un concepto difuso, desde el punto de vista neuroanatómico y funcional, por lo que niega la existencia de este y reconoce la escasa validez explicativa de la teoría con relación a la interpretación de los procesamientos emocionales. En los modelos defendidos por los autores encuadrados dentro de esta categoría de estudios fisiológicos y biológicos, se establece la existencia de estructuras cerebrales específicas responsables de la experiencia emocional y sentimental. Estos postulados servirán de base para el surgimiento de las nuevas investigaciones neurológicas en el ámbito de las emociones, y dan lugar a disciplinas como la neurociencia afectiva, cuyo objeto de estudio consiste en delimitar los fenómenos emocionales y sus correlatos neuronales.

De la fusión de ambas contribuciones, la teoría de James-Lange y Cannon- Bard, surge la novedosa aportación de los investigadores Schachter y Singer, para los cuales las emociones son elaboradas a partir de respuestas fisiológicas en las que la interpretación de los estímulos juega un papel vital. Así, defienden que el origen de las emociones depende, por un lado, de la interpretación e inferencias que realicemos a partir de las respuestas fisiológicas periféricas y, por otro lado, de la evaluación de la situación. Esto determina la intensidad de la emoción, algo muy importante en la evaluación de la situación y el resultado final. La forma de interpretar las respuestas periféricas condicionará la intensidad emocional como baja, media o alta y la evaluación la cualidad de la experiencia emocional, que daría lugar a la clasificación de las emociones: miedo, tristeza, alegría etc. (Plutchik, 1962). Según los investigadores cualquier estado emocional es el resultado de dos factores: el aurosal o activación fisiológica, y los aspectos cognitivos íntimamente relacionados con las causas de activación y el contexto: solo desde la interacción entre ambos puede llegar a desencadenarse la emoción.

Schachter y Singer dieron un nuevo giro a la investigación de la emoción, pues recuperaron el concepto evaluación, acercándose a los postulados cognitivos y sociales que seguirán algunos autores, como Richard Lazarus. Este abogaba por 
Núñez- Cansado, M., López López, A. y Vela Delfa, C.

Revisión teórico-cientifícia del marco conceptual de la emoción y el sentimiento y su aplicación al neuromarketing

defender que: "son las valoraciones cognitivas las que vinculan y median entre los sujetos y el ambiente, provocando emociones particulares como resultado de las evaluaciones específicas que realiza el sujeto, de su relación con el ambiente en virtud de su bienestar" (Citado en Melamed, 2016, p. 21). Estas aportaciones conducen a muchos investigadores a plantearse si la cognición es un requisito necesario para que se produzca la emoción.

Desde el ámbito de la psicología social, Zajonc, en el año 1980, rebate las hipótesis de Lazarus mostrando en sus investigaciones que muchas experiencias emocionales parecen producirse sin que intermedien aspectos cognitivos, entre ellas, el llanto de un niño. Considera que: "algunas reacciones afectivas pueden tener lugar fuera de conciencia y pueden influir sobre el propio procesamiento cognitivo pro-vías independientes, es decir, el planteamiento contempla la posibilidad de desvincular afectos de cogniciones" (Casado y Colomo, 2006, p. 10).

Comienza así un nuevo debate que abrirá el camino para la diferenciación entre la emoción, como proceso fisiológico con función básicamente adaptativa, y el sentimiento, como proceso cognitivo con fuertes influencias culturales. Consecuentemente, se produce un incremento de las investigaciones que indagan acerca de la naturaleza cultural y social de la emoción.

Paul Ekman, realiza estudios transculturales y evolutivos con el objeto de medir las emociones mediante el análisis de los correlatos autónomos de la expresión facial (Ekman \& Oster, 1981). Los investigadores defienden que las expresiones faciales de las emociones no están culturalmente determinadas, sino que son universales, tal y como advirtieron las teorías evolutivas. Así, llegó a desarrollar un Sistema de Codificación Facial de acciones (Facial Action Coding System), que permitía clasificar las expresiones del rostro humano. Con posterioridad, esta codificación sirvió de base para el diseño de gran parte del software empleado hoy en día en el estudio de las emociones en neuromarketing.

A raíz de los avances logrados desde la neurofisiología, se abre una nueva vía interpretativa en torno a la emoción, que la contempla como un proceso multidimensional en el que intervienen tanto los aspectos fisiológicos, como los cognitivos y evaluativos del sujeto -muy influenciados social y culturalmente-, claramente determinantes en la generación de los sentimientos, que pasan a comprenderse como subproductos de la emoción.

Kleinginna y Kleinginna defienden la emoción como:

Un complejo conjunto de interacciones entre factores subjetivos y objetivos, mediadas por sistemas neuronales y hormonales que: (a) pueden dar lugar a experiencias efectivas como sentimientos de activación, agrado desagrado; (b) generar procesos cognitivos tales como efectos perceptuales relevantes, valoraciones, y procesos de etiquetado; (c) generar ajustes fisiológicos...; y (d) dar lugar a una conducta que es frecuentemente, pero no siempre, expresiva, dirigida hacia una meta y adaptativa (Kleinginna \& Kleinginna, 1981, p. 355).

Vivat Academia. Revista de Comunicación. 2021, nº 154, 381-407 
Núñez- Cansado, M., López López, A. y Vela Delfa, C.

Revisión teórico-cientifícia del marco conceptual de la emoción y el sentimiento y su aplicación al neuromarketing

En la misma línea Scherer, profesor de psicología y director del Centro Suizo de Ciencias Afectivas en Ginebra, defiende que las emociones son fruto de cambios filogenéticos constantes, originados por procesos dinámicos, complejos y multifactoriales. Por lo tanto, para llegar a describir la emoción se requiere del análisis de los procesos que participan en ella, entre ellos, los procesos cognitivos, los neurofisiológicos, los motivacionales, los expresivos-instrumentales y de sentimiento, y estudiar cómo estos se correlacionan con diferentes sistemas orgánicos. Solo el análisis de los procesos en su conjunto podrá explicar el proceso emocional (Sherer, 1884). Así lo defendió el científico Gregorio Marañón en sus investigaciones sobre la emoción (Marañón, 1920a; Marañón 1920b), para la que mantenía que era necesario la interacción entre la actividad fisiológica y los procesos cognitivos. Si bien la activación fisiológica era una condición sine qua non, en ningún caso suficiente para que se produjera una emoción. Claramente, Marañón reivindicaba que, para que esta tuviera lugar, era necesario la valoración de los cambios corporales producidos tanto por la activación fisiológica como por el contexto.

A mediados del siglo $\mathrm{XX}$, los postulados teóricos aportados por el psicólogo cognitivista Mandler (1975) reafirman el papel de los procesos cognitivos en los procesos emocionales. La experiencia de la emoción y la conducta emocional son el resultado de la interacción entre la activación autonómica y la interpretación y valoración cognitiva: la activación reflejará la intensidad, mientras que los procesos cognitivos reflejarán la cualidad de la emoción. Esta interacción será la clave para diferenciar la emoción del sentimiento. De tal forma que, siguiendo las definiciones de Arnold (1960), las emociones son el resultado de una evaluación positiva o negativa, mientras que los sentimientos se originan en la interpretación más o menos de beneficiosa o perjudicial, que realiza el sujeto acerca de esa primera evaluación.

Desde este enfoque, la noción de emoción se moldea para ser entendida como un proceso fisiológico, que activa experiencias subjetivas consideradas sentimientos, en los que intervienen procesos cognitivos, que actúan como motivadores de la conducta y que están sujetos a la adaptación al entorno. Para los investigadores, describir todos los procesos implicados se convierte en primordial, a fin de poder analizar y revelar correctamente la emoción.

Desde la perspectiva fisiológica- biológica y cognitivista, la llegada de la teoría de los Marcadores Somáticos del neurocientífico Antonio Damasio (2006) ofrecerá un encuadre a los postulados que defienden la emoción como proceso multifactorial.

Según la hipótesis de los marcadores somáticos, las emociones influyen en nuestra percepción, en nuestra evaluación y, lo más importante, en las decisiones: racionalidad y emoción son dos elementos que actúan conjuntamente. Con esta teoría, las emociones se convierten en elementos clave en nuestra toma de decisión y en nuestra evaluación: nuestras decisiones requieren de una evaluación emocional, ya que no es posible tomar decisiones sin ese mecanismo. 
Núñez- Cansado, M., López López, A. y Vela Delfa, C.

Revisión teórico-cientifícia del marco conceptual de la emoción y el sentimiento y su aplicación al neuromarketing

Según la hipótesis del Marcador Somático, tenemos algunos marcadores que nos provocan una sensación de alarma sin que seamos conscientes de ello. Son un tipo especial de sentimientos, generados a partir de emociones secundarias, adquiridas mediante aprendizaje. Así, cuando tenemos que tomar una decisión, nuestro cerebro acude a los conocimientos y experiencias adquiridos en el trascurso de la vida. Esta búsqueda se traduce en reacciones somáticas, que se asocian a un estado emocional. La emoción conduce a la construcción de un conjunto de mapas neuronales en el organismo que pueden estar asociados a una configuración relacionada con la alegría y sus variantes; o con mapas que suponen la base del estado mental, que identificamos con la pena, el miedo, la angustia, la culpabilidad y/o la desesperación. El marcador somático sería una señal, en forma de sensación somestésica, que contribuye a optimizar nuestras decisiones y nuestro razonamiento.

Para Damasio es imprescindible diferenciar sentimiento de emoción o: "sentimiento de sentimiento de emoción" (Damasio, 2006). Así la emoción es entendida como una reacción reguladora de cambios corporales, se trata de "un conjunto complejo de respuestas químicas y neuronales que forman un patrón distintivo" (Damasio, 2003, p. 65). La evaluación de los cambios corporales sería imprescindible para considerar la aparición de los procesos emocionales; pero, a diferencia de otros autores, hace referencia al plano en el que se realiza la evaluación. Tal y como defiende, no siempre es necesario que la evaluación se realice en el plano consciente, sino que puede producirse de forma inconsciente. No en vano, el autor define el término deseo como: "sentimientos conscientes de tener un apetito y a la eventual consumación o frustración de dicho apetito" (Damasio, 2003).

En este marco conceptual, los sentimientos son definidos desde la percepción y la evaluación; así, un sentimiento es "la percepción de un determinado estado del cuerpo, junto con la percepción de un determinado modo de pensar y de pensamiento con determinados temas" (Damasio, 2003). Por ello es importante tener en cuenta que, para el autor, los sentimientos no son solo origen de la interpretación y evaluación de las emociones sino también de cualquier cambio homeostático. La esencia de ambos conceptos es distinta: los sentimientos se encuentran en el plano de los pensamientos (en cómo estos representan al cuerpo ante un estado reactivo) mientras que las emociones son resultados inmediatos, automáticos, es decir, son cambios corporales que generan estados reactivos que buscan situar al organismo en circunstancias propicias para la supervivencia y el bienestar. Por tanto, sentimientos y emoción forman parte de un proceso imprescindible para comprender las funciones de supervivencia en el ser humano.

Así, se alcanzan puntos de encuentro entre las distintas perspectivas: la biológica, la fisiológica y la psicológica. Todas ellas defienden una visión multifactorial de las emociones y reivindican la necesidad de diferenciar emoción y sentimiento.

Otras disciplinas, como la antropología y la sociología, van a mostrar también el valor de los sentimientos como elementos de interpretación de las emociones, al incluir nuevos factores imprescindibles para la descripción de la emoción. Esta se 
Núñez- Cansado, M., López López, A. y Vela Delfa, C.

Revisión teórico-cientifícia del marco conceptual de la emoción y el sentimiento y su aplicación al neuromarketing

describe como "producto del interjuego entre una semántica (cultura visual, escrita, oral) y el individuo; y de las relaciones entre individuos a mayor o menor escala" (García Andrade, 2019, p. 44). Igualmente, como puede observarse, estas aportaciones rompen con el reduccionismo fisiológico de las emociones propio de las primeras teorías, y se inclinan por una concepción del término condicionada por las influencias de carácter sociocultural. No obstante, desde estas disciplinas se acepta la orientación del paradigma de la neurociencia, pero se critica el exceso de individualismo presente en sus definiciones. De ahí que, incidan en el poder de la sociedad y las culturas en el proceso emocional individual. Frente al innatismo de las emociones, este punto de vista defiende la influencia del entorno sobre su aprendizaje y se acerca a la definición de marcadores somáticos de Damasio: "como una herramienta de aprendizaje emocional/corporal/ cerebral en nuestra biografía" (García Andrade, 2019). Así, destacan que las experiencias individuales, generadas a partir de estos marcadores somáticos, están determinadas por contextos sociales. No se trata de disciplinas contrapuestas en relación con el concepto biológico y fisiológico de la emoción, sino complementarias. Tal y como muestran las aportaciones realizadas desde el paradigma de la neurociencia, como es la del neurocientífico Joseph LeDoux, director del Emotional Brain Institute, quien reconoce que las emociones tienen una fuerte carga cultural; si bien centra los detalles de su teoría en la distinción entre emociones y esquemas emocionales. Establece, que estos últimos son fruto de construcciones sociales, realizadas por el individuo en base a su biografía y entorno (LeDoux, 2002).

\subsection{Las emociones y los sentimientos en el marketing}

Tanto desde la neurociencia como desde la psicología, la sociología y la antropología, parece haber cierto consenso a la hora de reconocer la función de la emoción en la toma de decisiones y en los procesos de razonamiento (Scheutz, 2000; Ahvenniemi et al. 2018; Russell, 1980; Lewinski et al. 2014).

Esta nueva visión en torno a la funcionalidad de la emoción, la convierte en objeto de estudio de disciplinas como el marketing, la publicidad, la comunicación o la economía. De este modo, comienzan a surgir aportaciones imprescindibles para comprender la relación entre la emoción y el comportamiento del consumidor.

Poels y Dewitte (2006) concluyen en sus estudios que la emoción es un predictor de la eficacia publicitaria, y consideran que la medición de la respuesta emocional del consumidor proporciona una evaluación sólida del impacto que un determinado anuncio tendrá en su actitud, en su comportamiento y en su recuerdo. Por su parte, el equipo del Dr. Page (1990) encontró que la memorabilidad de un anuncio emocional era más acentuada que la de uno informativo. Por estas razones, los investigadores de este ámbito comienzan a aceptar el peso de las emociones en la toma de decisiones y tratan de analizarlas para comprender mejor a su consumidor.

La investigación de la emoción como elemento clave para comprender al consumidor no es algo nuevo, ya en 1924, cuando el psicólogo Watson fue contratado

Vivat Academia. Revista de Comunicación. 2021, nº 154, 381-407 
Núñez- Cansado, M., López López, A. y Vela Delfa, C.

Revisión teórico-cientifícia del marco conceptual de la emoción y el sentimiento y su aplicación al neuromarketing

como vicepresidente en Walter Thompson, introdujo en la agencia la investigación de mercados como prueba científica de la respuesta esperada en el target. Para él este era un requisito indispensable para inducir comportamientos en los compradores, acordes con los objetivos de marketing.

No en vano, Watson creó diversas campañas en torno a los resultados de sus investigaciones en relación con tres emociones básicas: miedo, ira y amor. Partía de una concepción innata de las emociones y del supuesto de que es posible condicionarlas mediante estímulos neutros, como marcas o productos.

Es obvio, que el rol de la emoción como elemento clave en la toma de decisión del consumidor no es un concepto nuevo, ni amasado en el reciente siglo XXI. Sin embargo, desde las primeras investigaciones de Watson hasta la actualidad, existen grandes cambios que han sido impulsados tanto por los avances en el estudio del funcionamiento de nuestro cerebro como por la aplicación de las técnicas de neurociencia y el big data. Estas han supuesto una importante revolución en la investigación en marketing.

Esta es la oportunidad de acercarse, tal y cómo defendía Watson, a un conocimiento más eficaz y científico del comportamiento del consumidor. Un valioso conocimiento, que hace posible al anunciante desplegar estrategias más eficaces con las que posicionar mejor a sus marcas e incrementar sus beneficios.

A comienzos del siglo XXI, se produce un auge de la literatura científica sobre la aplicación de los conceptos de emoción y sentimiento al marketing y al neuromarketing; aunque pocos estudios han reproducido de forma rigurosa hallazgos empíricos sobre el tema (Lim, 2018) o han aportado nuevos marcos teóricos. En realidad, estos trabajos representan todavía un porcentaje muy bajo en el conjunto de las publicaciones.

La revisión bibliográfica (de 2002 a 2013) acerca de los estudios de la emoción desde la perspectiva del marketing realizada por el equipo del Dr. Gaur determinó que tan solo 340, de un total de 14.321 artículos, abordaban este tema o, lo que es lo mismo, un 2,37\% del corpus analizado (Gaur et al. 2014). Si bien, este bajo índice de publicaciones podría estar ligado a la complejidad que implica estudiar las emociones, tanto a la hora de reconocerlas, como de definirlas, medirlas e interpretarlas, los autores notifican cierto interés al alza sobre el tema; lo que reflejaría un aumento de los trabajos publicados, que alcanzaron su pico máximo durante 2009, 2010 y 2011.

A diferencia de lo que ocurre en el ámbito de la psicología, centrado más en las correlaciones con otros procesos como la atención, la toma de decisiones, la memoria y la descripción de un marco teórico (Hamelin et al., 2017), las investigaciones sobre las emociones en el marketing se han fijado, sobre todo, en aspectos relacionados con la métrica y sus aplicaciones al análisis del consumidor. 
Núñez- Cansado, M., López López, A. y Vela Delfa, C.

Revisión teórico-cientifícia del marco conceptual de la emoción y el sentimiento y su aplicación al neuromarketing

Ha sido con el tiempo, que la definición de la emoción desde el plano de la inconsciencia, como un proceso automático, ha ido cobrando importancia. Por esta razón, los investigadores han tenido que desarrollar técnicas que permitan analizar estos mecanismos inconscientes. Así, progresivamente, se ha ido aplicando la tecnología de la neurociencia al estudio la comunicación y del marketing, hasta el punto de generarse campos interdisciplinares como el neuromarketing o la neurocomunicación.

Precisamente, la influencia que tienen las emociones en nuestros comportamientos, en nuestros pensamientos, o en la toma de nuestras decisiones (Carlson et al., 2007), es lo que, en los últimos años, está despertando un gran interés por medir y conocer las estructuras del cerebro responsables de los procesos emocionales entre la comunidad científica. De esta forma, en cierto modo, se dejan de lado los supuestos de los paradigmas cognitivos e interpretativos para dar un mayor peso a los fisio-biológicos, y, también, a que en la investigación sobre la emoción se prioricen los aspectos metodológicos por encima de los conceptuales.

En este punto, comienzan a perfilarse claras diferencias entre los conceptos de emoción y sentimiento. Así, los sentimientos son analizados, básicamente, desde la perspectiva del big data, mientras que las emociones dejan de analizarse con métricas basadas en test clásicos, como el Self Assement Manikin o el PrEmo, para ser medidos con herramientas y tecnologías propias de la neurociencia. En los últimos años, sin embargo, ambas perspectivas buscan interrelacionarse para crear metodologías mixtas útiles en la investigación de ambos conceptos, aunque en la literatura científica este enfoque es todavía muy residual (Kumar, 2019).

Las investigaciones sobre el análisis de los sentimientos en el ámbito del marketing surgen con fuerza a partir de principios del siglo XXI. Estas se han ocupado de analizar y aplicar métricas de sentimientos, principalmente en el entorno digital. Sin embargo, no se ha encontrado ninguna publicación que se centré en la investigación de este concepto en términos teóricos, bien desde descripciones, bien desde definiciones, bien desde la identificación de las funciones o los fundamentos psicofisiológicos de los sentimientos. Principalmente, el ámbito de estudio conocido como minería de sentimientos está limitado en la literatura científica a la búsqueda de métodos para identificar, extraer y analizar sistemáticamente las opiniones, actitudes y sentimientos de las personas, a partir de una gran cantidad de datos textuales (Pang \& Lee, 2004). De tal manera que se pueden identificar dos modalidades de métricas sobre la investigación de los sentimientos: aquellas que utilizan los enfoques basados en léxicos y aquellos que utilizan las redes de aprendizaje.

Los enfoques basados en léxicos asumen que un conjunto de palabras claves, en un ámbito particular, marcan el sentimiento del texto al completo (Kumar et al, 2019; Yao \&Wang 2020; Hamilton et al., 2016; Sun et al., 2020). Esta metodología necesita del diseño de algoritmos muy complejos, como los modelos de clasificación NB (Naives Baynes) o SVC (Modelos de vectores de soporte). Por su parte, las 
Núñez- Cansado, M., López López, A. y Vela Delfa, C.

Revisión teórico-cientifícia del marco conceptual de la emoción y el sentimiento y su aplicación al neuromarketing

metodologías basadas en redes de aprendizaje analizan las representaciones de datos existentes para extraer predicciones en relación a un conjunto de datos (Wilson et al., 2005; Neethu \& Rajasree, 2013; Shapiro et al., 2020). Algunos estudios han integrado los enfoques léxicos y las redes neuronales a través de mecanismos de atención (Lei et al., 2018). Aunque, como se mencionó anteriormente, en la literatura científica apenas existen referencias al respecto. La mayor parte de los estudios sobre la métrica de los sentimientos utilizan como corpus principal las redes sociales para estudiar temas muy dispares como el sentimiento en el consumo de energía (Sun et al., 2020); las respuestas sentimentales de consumidores ante catástrofes naturales (Yao \&Wang 2020); la satisfacción en el ámbito del turismo (Henríquez, 2016) o de la banca (Angulo Murillo, 2020); y para el análisis de los sentimientos vinculados a la política (Arcila-Calderón et al., 2017; Romero Moreno et al. 2020) etc. En los últimos meses, se han disparado los estudios de minería de sentimientos en torno a temas relacionados con la reacción de la población ante la crisis sociosanitaria de la COVID (García y Berton, 2021; Alamoodi et al., 2020). Este gran interés queda reflejado en el incremento de artículos científicos en la materia, sobre todo desde el área del marketing.

En relación con las investigaciones metodológicas sobre la emoción dentro del ámbito del marketing, los artículos revisados muestran un creciente interés por analizar el poder de las emociones en la toma de decisiones del consumidor. En ellas se parte de una concepción de la emoción desde el paradigma fiosio-biológico y evolutivo. Así, se pretende analizar el correlato fisiológico que es activado por las emociones, que permita descifrar el comportamiento del consumidor. Para analizar las emociones se utilizan, principalmente, dos tipos de herramientas: aquellas en las que se registra la actividad metabólica del cerebro, y las que reconocen su actividad eléctrica.

Dentro de las métricas que parten de datos obtenidos de registros metabólicos de la actividad del cerebro, cobran especial relevancia las obtenidas mediante técnica de FMRI (Morin, 2011; Zurawicki, 2010; Rampl et al., 2016; Plassmann et al., 2008; Ruanguttamanun, 2014; Cabeza \& Nyberg, 2000). Mediante su uso se intentan recuperar imágenes de estructuras cerebrales involucradas en la decisión de compra, como el Nucleus Acumbens, la Ínsula, el Cortex Prefontal Ventromedial o el Córtex Cingulado Anterior; en otras palabras, comprobar cómo estas responden ante estímulos del marketing. Pero su alto coste y escasa validez ecológica, ha hecho que este tipo de estudios quede relegado a centros de alta investigación y sean inaccesibles para la gran mayoría de las empresas de neuromarketing (NúñezCansado et al. 2020), que terminan optando por medios más económicos y flexibles mejor adaptados a la movilidad del consumidor en situación experimental. Estas alternativas se centran sobre todo en la toma de registros de la actividad eléctrica cerebral (EEG) (Vecchiato et al., 2014; Hakim et al., 2019; Hsu \& Chen, 2020; GolnarNick et al., 2019; Gill \& Singh, 2020; Bastiaansen et al., 2018; Hajcak et al., 2012; Olofsson et al., 2008) y de la actividad electrodérmica o respuesta galvánica de la piel (GSR) (Avinash et al., 2018; Groeppel-Klein, 2005; Baldeesh \& Senior, 2008; Aiger et al., 2013; Martínez Herrador et al., 2020). Su principal objetivo es analizar los 
Núñez- Cansado, M., López López, A. y Vela Delfa, C.

Revisión teórico-cientifícia del marco conceptual de la emoción y el sentimiento y su aplicación al neuromarketing

correlatos fisiológicos asociados a las distintas emociones ante los estímulos del marketing.

La descripción de las emociones, como correlatos fisiológicos, ha sido complementada por parte de posturas más cercanas a los paradigmas biologicistas que aceptan la función social y universal de las emociones y su correlación con expresiones faciales. Esto ha permitido desarrollar softwares, como el Face Reader, basado en la metodología sobre expresiones faciales propuesta por Paul Ekman \& Friesen (1971): Facial Action Coding System. Como se sabe, estos programas parten de la premisa de que existe una correlación entre ciertas expresiones faciales específicas y las seis emociones básicas (Azacarate et al., 2005; Lewinski et al., 2014; Salah et al., 2009; Wahab et al., 2015; Chen et al., 2013). Estas correlaciones suponen una fuente de información sobre los patrones existentes entre la activación muscular y las emociones.

No obstante, existen otras técnicas menos populares que intentan analizar los correlatos fisiológicos como, por ejemplo: el electromiograma, el electrocardigrama, etc. Todas ellas interpretan la emoción desde la perspectiva neurofisiológica. Sin embargo, su análisis ocuparía por sí solo una publicación completa y exclusiva, que en este trabajo no es posible contemplar.

\section{UN PUNTO DE INFLEXIÓN: LAS TEORÍAS DE LA EMOCIÓN Y EL SENTIMIENTO Y SU APLICACIÓN AL NEUROMARKETING}

Una adecuada revisión, delimitación y actualización del marco conceptual teóricocientífico de la emoción y el sentimiento es imprescindible para evitar los posibles malentendidos que, en los diferentes ámbitos de investigación, su aplicación podría conllevar. En los últimos años, los programas de investigación científica tienden a romper con la concepción teórica primigenia y tradicional sobre la emoción y el sentimiento, que unificaba la identificación de ambos conceptos (Scarantino, 2018), para adoptar una perspectiva de estudio multifactorial y diferencial sobre ambos. Sin embargo, en el lado del neuromarketing y su praxis, esta investigación pone de manifiesto carencias referentes a la aplicabilidad del enfoque multifactorial defendido en el ámbito teórico-científico, al comprobar como los mecanismos psicofiosiológicos de los sentimientos y la influencia de los procesos cognitivos en las emociones son ignorados en la práctica profesional en múltiples ocasiones. De esta manera, los sentimientos quedan relegados a evaluaciones frecuentemente basadas en análisis léxicos y, en el mejor de los casos, combinados con metodologías IA (Inteligencia Artificial). La investigación del concepto de emoción se fundamenta básicamente en el estudio de los correlatos neurofiosiológicos de los procesos emocionales, dejando a un lado la diferencia conceptual y funcional entre emoción y sentimiento, así como la necesidad de interrelación entre ellos.

La emoción es un conjunto de respuestas neuroquímicas y hormonales, una respuesta orgánica, que nos predispone a reaccionar de una determinada forma ante un estímulo externo, provocando una conducta somática. Tomar consciencia de estos 
Núñez- Cansado, M., López López, A. y Vela Delfa, C.

Revisión teórico-cientifícia del marco conceptual de la emoción y el sentimiento y su aplicación al neuromarketing

cambios permite activar procesos cognitivos, que facilitan una percepción global e integrada, y una evaluación de esa emoción a partir de una interpretación consciente mediante nuestros pensamientos. La mente tiene pensamientos y emociones, por lo que el estudio de unos excluyendo los otros nunca será totalmente satisfactorio (LeDoux, 2002). Las emociones surgen al tratar de interpretar los estímulos externos. Los sentimientos aparecen al tratar de interpretar las emociones; interpretación regulada por nuestros pensamientos, lo que supone que los sentimientos son asociaciones mentales. Una misma emoción puede dar lugar a diferentes sentimientos, según la interpretación y el análisis del sujeto. Por tanto, medir únicamente una activación emocional puede conducir a numerosas confusiones en la difusión de resultados. Definitivamente, son dos procesos que interactúan y que se necesitan mutuamente. Los sentimientos necesitan de la activación emocional, y las emociones, dado su carácter fugaz, requieren de los sentimientos para prolongar el impacto de su activación en el tiempo y llegar a afectar de manera permanente, tanto atención como emoción. No en vano, tal y como describe LeDoux (2002), el sentimiento requiere acceder a una serie de informaciones previas que se actualizan en lo que se denomina "memoria a corto plazo" o "memoria de trabajo". Ambas interactúan para generar mapas cognitivos, que serán los responsables en nuestra toma de decisiones. Sin embargo, en la literatura del neuromarketing la emoción ha tomado un papel mucho más relevante, haciendo que los sentimientos queden relegados a un papel secundario e, incluso, llegando a confundir ambos procesos, tanto desde el punto de vista conceptual como de la propia praxis. Sin embargo, muy probablemente, el sentimiento sea, más que la emoción, el responsable de la mayor parte de nuestros comportamientos como consumidores. En consecuencia, el denominado marketing emocional debiera definirse como iun marketing sentimental? Dado que el objetivo de toda marca no es solo lograr una activación fisiológica sino un sentimiento que se prolongue en el tiempo, la relación entre consumidor y marca ha de ser sentimental. La activación de una emoción no siempre lleva consigo la aparición de un sentimiento $\mathrm{y}$, sin embargo, es el sentimiento el que nos lleva a la fidelización del cliente. Fue Marc Gobé quien acuñó en el año 2001 el término de marketing emocional como una disciplina del marketing, cuyas estrategias tienen como principal objetivo lograr un vínculo afectivo entre la marca y sus usuarios, consumidores y potenciales clientes, para que estos sientan la marca como algo propio y necesiten ser parte de ella. Para lograr un vínculo afectivo es crucial el sentimiento, por lo que el término más adecuado sería el del "marketing sentimental".

Además de desvirtuar el marco conceptual, el hecho de confundir estos dos términos nos puede inducir a error a la hora de realizar la evaluación y descripción del diseño de la investigación en marketing, lo que afectaría gravemente a su validez. Ambas circunstancias pueden ser parte de la justificación que esgrimen algunos estudios contrarios a la práctica del neuromarketing. Así, ciertos autores concluyen que, si bien la actividad cerebral indudablemente tiene manifestaciones neurológicas y fisiológicas, existe un creciente cuerpo de evidencia, obtenida de varios metaanálisis, que desafía la hipótesis de que las medidas fisiológicas se corresponden con instancias particulares de emociones (Lindquist et al., 2012; Clark-Polner et al.,

Vivat Academia. Revista de Comunicación. 2021, nº 154, 381-407 
Núñez- Cansado, M., López López, A. y Vela Delfa, C.

Revisión teórico-cientifícia del marco conceptual de la emoción y el sentimiento y su aplicación al neuromarketing

2017; Barret, 2018). En la actualidad, no existe un consenso sobre qué medidas psicofisiológicas representan mejor las diferentes emociones.

En verdad, es importante ser muy cautos para evitar errores irreversibles que dañen la disciplina, impidiendo que prospere. Normalmente, y en líneas generales, los deslices o fallos cometidos se deben a la falta de validez en el constructo o grado de correspondencia entre las variables observadas y el constructo teórico que se quiere medir. Así, por ejemplo, el engagement constituiría un índice de sentimiento, no de emoción. Sin embargo, los resultados relativos a este índice se producen por la medición de la activación emocional con metodologías como GSR. De ahí la importancia de diseñar la investigación y el uso de las distintas metodologías de acuerdo con el constructo que se va a medir. Tal y como afirma Damasio, las metodologías de medición de sentimientos mostraban que la conductancia dérmica precedía siempre a la señal que indicaba que se estaba produciendo un sentimiento (Damasio, 2003). Sin embargo, no reflejaba la esencia del sentimiento, ya que los sentimientos van más allá de una mera manifestación fisiológica en una estructura concreta, y requieren de la cartografía de innumerables aspectos de los estados corporales en las regiones sensoriales, diseñadas para recibir señales procedentes del cuerpo. El proceso no está en un solo área cerebral (Damasio, 2003). Las valencias de los sentimientos parecen estar relacionadas con la desactivación o activación de la corteza prefrontal; activación, en el caso del sentimiento de felicidad, y desactivación en el sentimiento de tristeza. Sin embargo, en múltiples estudios, esta desactivación se interpreta como falta de atención o procesamiento y no como una valencia negativa de los sentimientos. Los resultados de valencias sentimentales no pueden ser evaluados mediante GSR, por lo que a menudo, se utiliza la metodología de análisis facial. Sin embargo, posiblemente debido a esa falta de validez en el constructo, son muchos los estudios que muestran que existe un desfase en el resultado de la valencia del sentimiento. Así, por ejemplo, Férnandez-Dols (1995), en una investigación realizada con el objetivo de distinguir las teorías que argumentaban que las expresiones emocionales reflejaban sentimientos, de las teorías que defendían que expresaban actos comunicativos -con relación a los ganadores de las medallas de oro en los Juegos Olímpicos-, concluyó que los atletas ganadores no sonrieron cuando se enteraron de que habían ganado, sino al volverse hacia la audiencia. A partir de esto, concluyeron que las expresiones sirven como una señal social, más que como un marcador de emoción.

Además, la mayoría de las metodologías del neuromarketing se basan en el monitoreo de la respuesta durante los primeros segundos tras la presentación del estímulo, por lo que no se debe olvidar que el procesamiento de la información relativa a los sentimientos sucede en otras escalas temporales, ya que implica procesos cognitivos superiores más allá de la respuesta inmediata y automática. Estos procesos pueden suponer la clave en la toma de decisiones a largo plazo. La falta de validez concurrente y predictiva se produce al desatender estas circunstancias. Respecto a la validez concurrente, tanto la medición como la comprobación del criterio se llevan a cabo en distinto momento, lo que impide relacionar ambos procesos para valorar el estado actual del sujeto. En el caso de la 
Núñez- Cansado, M., López López, A. y Vela Delfa, C.

Revisión teórico-cientifícia del marco conceptual de la emoción y el sentimiento y su aplicación al neuromarketing

validez predictiva, que permite establecer predicciones con respecto al comportamiento, se vulnera cuando el uso de los instrumentos y los criterios definidos no atienden a las diferencias existentes entre ambos conceptos.

Sería necesario utilizar una metodología mixta que permita el análisis de la evaluación de los sujetos en un plazo mayor al habitualmente utilizado en neuromarketing. Hay que tener presente que los sentimientos son evaluaciones que pueden realizarse tanto de forma consciente e inconsciente. Tomando solo la referencia de parámetros psicofisiológicos, en un momento puntual y a nivel inconsciente, sobre un estímulo determinado, se dejan de lado factores vitales en la formación del sentimiento como la influencia cultural, social, los estilos cognitivos y las experiencias. De esta forma, es posible conocer tanto la activación emocional como la interpretación que de ella hace el sujeto para reconstruir sus dimensiones semánticas, relacionadas no solo con el sentimiento sino también con otros datos predictores muy importantes en la toma de decisiones. Esta lectura es crucial para comprender el comportamiento y su durabilidad, más allá de la activación emocional que lo desencadena.

Por todo esto, es importante realizar un exhaustivo trabajo teórico y experimental, que ayude a explicar adecuadamente los constructos que activan las emociones y los sentimientos.

\section{CONCLUSIONES}

La aplicación de técnicas de neuromarketing debe comportar un mayor rigor científico, lo que implica no solo realizar interpretaciones teóricas más correctas, que partan de un marco conceptual actualizado, sino aplicar metodologías mixtas e integradas que sirvan para medir y observar la actividad cerebral, siendo conscientes siempre de sus limitaciones. Aunque los expertos en neurociencia han avanzado mucho a la hora de medir la actividad cerebral, estamos muy lejos de la descodificación y la extracción de los pensamientos que forjan los sentimientos del sujeto y que, a su vez, determinan la toma de decisión. Es necesario reflexionar sobre lo que estamos midiendo y sus implicaciones pues, a menudo, las técnicas empleadas tienen características diferenciales que limitan el campo de especulación.

La búsqueda de las evidencias con relación a la investigación del consumidor está excediendo, en muchos casos, la relación entre mente y cerebro. Conviene partir de teorías dinámicas, que analizan el cerebro como un sistema complejo con múltiples interacciones recíprocas, y de un concepto más integral y multifactorial de los procesos emocionales. Asimismo, las metodologías deben ajustarse a la naturaleza de los procesos y atender a los límites de los posibles resultados mediante un método científico capaz de transmitir adecuadamente los avances. Desatender estas limitaciones puede conducirnos a subestimar o sobreestimar los hallazgos neurocientíficos y cometer reduccionismos que, no en vano, a veces se utilizan como gancho para generar nuevas oportunidades de negocio, sin valorar el grave perjuicio para el buen hacer y devenir de esta disciplina. Ejemplos de ello son afirmaciones 
Núñez- Cansado, M., López López, A. y Vela Delfa, C.

Revisión teórico-cientifícia del marco conceptual de la emoción y el sentimiento y su aplicación al neuromarketing

tales como las encontradas en un análisis de páginas web relacionadas con el neuromarketing: "el $95 \%$ de nuestras decisiones son tomadas con las emociones"; "Monitorea y registra instantáneamente cómo los cerebros de los consumidores procesan lo que ven, escuchan y leen de un momento a otro"; " "fMRI ... permite a los investigadores ver la mente humana en tiempo real, ya que las emociones y los pensamientos cognitivos están en juego" (Lim, 2018. p. 139).

Hay que ser conscientes de que mientras no existan técnicas que posean una resolución espacial y temporal perfecta o, al menos, funcionen en torno a los ritmos propios y característicos de la actividad cerebral, las conclusiones obtenidas estarán sesgadas. En no pocas ocasiones se está sobrevalorando el poder del neuromarketing. Leer respuestas fisiológicas no implica tener acceso a nuestros pensamientos $\mathrm{y}$, con ello, a nuestros sentimientos, tan claves en la relación del consumidor con la marca. Esta imagen errónea sobre el súper poder asociado a estas metodologías ha conducido a crear una visión desfigurada del consultor de neuromarketing, creyéndole capaz de manipular nuestros pensamientos cuando, realmente, no existe un botón de compra, ni un consumidor zombi. Esto no quiere decir que el neuromarketing no constituya un avance importantísimo para el estudio del consumidor, sino que se ha de ser tener una conducta más cauta, responsable y ética, tanto en la interpretación de los resultados como en su difusión. Es muy importante no perder de vista que estamos entregando informes que incluyen interpretaciones que refieren una aproximación sobre el funcionamiento de los procesos del cerebro.

Es imprescindible adoptar una visión más integradora, metodológica y teóricamente, a la hora de investigar en neuromarketing, desde postulados que huyan del neurocentrismo y definan las emociones y los sentimientos atendiendo a su plano multifactorial. Esto es fundamental para alcanzar la confiabilidad y validez científica necesarias para que el neuromarketing se constituya en un área de conocimiento completa, respetada y fiable.

\section{REFERENCIAS}

Ahvenniemi H., Pennanen K., Knuuti A., Arvola A. \& Viitanen K. (2018). Impact of infill development on prices of existing apartments in Finnish urban neighbourhoods. International Journal of strategic property management, 22 (3) 157167. https:// doi.org/10.3846/ijspm.2018.1540

Aiger, M., Palacín M. y Cornejo J. M. (2013). La señal electrodérmica mediante Sociograph: metodología para medir la actividad grupal. International Journal of Social Psychology, (28), 3. https://doi.org/10.1174/021347413807719102.

Alamoodi A. H., Zaidan B. B., Albahri K. I. y Zaidan A. A. (2020). Sentiment analysis and its applications in fighting COVID-19 and infectious. Expert Systems With Applications, 114155. https://doi.org/10.1016/j.eswa.2020.114155. 
Núñez- Cansado, M., López López, A. y Vela Delfa, C.

Revisión teórico-cientifícia del marco conceptual de la emoción y el sentimiento y su aplicación al neuromarketing

Angulo Murillo, N. S. (2020). Modelo para el análisis de sentimientos del banco de encuestas con preguntas sobre coronavirus de la OMS empleando principios de minería de textos. Mikarimin, 31-39.

Arcila-Calderón C., Ortega-Mohedano F., Jiménez-Amores J. y Trullenque, S. (2017). Análisis Supervisado de sentimientos políticos en español, clasificación en tiempos real de Tweets basado en aprendizaje automático. el Profesional de la Investigación, 1699-2407.

Arnold, M. (1960). Emotion and Personality. Vol 1. Psychological Aspects. Columbia University Press.

Avinash T., Dikshant L. y Seema, S. (2018). Methods of Neuromarketing and Implication of the Frontal Theta Asymmetry induced due to musical stimulus as choice modeling. International Conference on Computational Intelligence and Data Science (ICCIDS 2018), 55-67. Gurgaon. India.

Azcarate, A., Hageloh, F., Sande, K. y Valenti, R. (2005). Automatic facial emotion recognición. https://bit.ly/2NihIBK

Baldeesh, G. y Senior, C. (2008). Examining the influence of fame in the presence of beauty : an electrodermal 'neuromarketing' study. Journal of Consumer Behaviour, (7), 4-5. https://doi.org/10.1002/cb.255.

Bagozzi R., Gopinath, M., y Nyer, P. (1999). El papel de las emociones en el marketing. Revista de la Academia de Ciencias del Marketing., (27)2, 184-206.

Barret, L. (2018). Emotion fingerprints or emotion populations ? A meta-analytic inves-tigation of autonomic features of emotion categories. Psychological Bulletin, $144,343-393$.

Bastiaansen M., Straatman S., Driessen E., Mitas O. y Stekelenburg Lin Wang, L. (2018) My destination in your brain: A novel neuromarketing approach for evaluating the effectiveness of destination marketing. Journal of Destination Marketing $\mathcal{E}$ Management, 76-88.

Bisquerra, R. (2000). Educación emocional y bienestar. Ciss- Praxis.

Cabeza, R. y, Nyberg, L. (2000). Imaging cognition II: An empirical review of 275 PET and fMRI studies. Journal of Cognitive Neuroscience, 1-47.

Carlson N. R., Heth D. C., Miller, H., Donahoe, J. W., Buskist, W. y Martin N. G. (2007). Psychology: The Science of Behavior. Pearson. 
Núñez- Cansado, M., López López, A. y Vela Delfa, C.

Revisión teórico-cientifícia del marco conceptual de la emoción y el sentimiento y su aplicación al neuromarketing

Casado, C. y Colomo, R. (2006). Un breve recorrido por la concepción de las emociones en la filosofía occidental. A Parte Rei. Revista de filosofía, (47)1-10.

Chen, J., Ariki, Y. y Takiguchi, T. (2013). Robust facial expressions recognition using 3D average face and ameliorated Adaboost. 21st ACM International Conference on Multimedia. 661-664. https:// doi.org/10.1145/2502081.2502173)

Clark-Polner, E., Johnson, T. y Barret, L. F. (2017). Multivoxel pattern analysis does not provide evidence to support the existence of basic emotions. Cerebral Cortex, 1944-1948.

Damasio, A. (2003). En busca de Spinoza. Ediciones Destino. S.A

Damasio, A. (2006). El error de Descartes. Crítica.

Díaz Ortíz, A. (2010). Teorías de las motivaciones. Innovación y experiencias educativas, $1-6$.

Dixon, T. (2003). From Passions to emotions. The Creation of a Secular Psychological Category. Cambridge University Press.

Ekman, P. y Friesen W.V. (1971). Constants across the cultures in the face and emotion. Journal of Personality and Social Psychology, 124-129.

Ekman, P. y Oster, H. (1981). Facial expressions of emotion. Studies in Psychology, 2 :7, 115-144.

Ekman, P. (1992). An argument for basic emotions. Cognition E Emotion, 169-200.

Fernández, A., Dufey, M. y Mourgues, C. (2007). Expresión y reconocimiento de emociones: un punto de encuentro entre evolución, psicofisiología y neurociencias. Revista Chilena de Neuropsicología, (2) 1, 8-20.

Fernández-Dols, J. M y Ruiz- Belda, M. A. (1995) ¿Son las sonrisas un signo de felicidad ? Revista de Personalidad y Psicología Social. (6), 113-119

García Andrade, A. (2019). Neurociencia de las emociones: la sociedad vista desde el individuo. Una aproximación a la vinculación sociología-neurociencia. Sociología, 39-71.

García, K. y Bertón L. (2021). Topic detection and sentiment analysis in Twitter content related to COVID-19 from Brazil and the USA. Applied Soft Computing, (101) 10757.

Gaur S., Herjanto H. y Makkar M. (2014). Reviewofemotionsresearchinmarketing, 2002-2013. Journal of Retailing and Consumer Services, 917-923. 
Núñez- Cansado, M., López López, A. y Vela Delfa, C.

Revisión teórico-cientifícia del marco conceptual de la emoción y el sentimiento y su aplicación al neuromarketing

Gill, R. y Singh, J. (2020). Study of neuromarketing techniques for proposing cost effective information driven framework for decision making. Materials Today: Proceedings. https://doi.org/10.1016/j.matpr.2020.08.730.

Golnar-Nick, P., Farashi S. y Safari M. S. (2019). The application of EEG power for the predictión an interpretación of consumer decisión-making: a neuromarketing $\begin{array}{lllll}\text { study. } & \text { Physiology } & \text { B } & \text { Behavior, }\end{array}$ https://doi.org/10.1016/j.physbeh.2019.04.025.

González, B. I. (2017). "En busca de Spinoza", de Antonio Damasio. Culturasmas: https://bit.ly/2ZxdXLb

Groeppel-Klein, A. (2005). Arousal and consumer in store behavior. Brain Research Bulletin, 428-437.

Hajcak G., Weinberg A., MacNamara A. y Foti D. (2012). ERPs and the study of emotion. Oxford University Press.

Hakim, A., Klorfeld, S., Sela, T., Friedman, D. y Shabat-Simon, M. (2019). Machines learn neuromarketing: Improving preference. International Journal of Research in Marketing. https://doi.org/10.1016/j.ijresmar.2020.10.005.

Hamelin, N., Moujahid, O. y Thaichon O. (2017). Emotion and advertising effectiveness: A novel facial expression analysis approach. Journal of Retailing and Consumer Services, 36, 103-111.

Hamilton, W. L., Clark, K., Leskovec, J. y Jurafsky, D. (2016). Conference on empirical methods in natural language processing. Classification of domain-specific feelings by merging knowledge of feelings from multiple sources. 595-605.

Henriquez Miranda, C. G. (2016). Minería de Opiniones basado en la adaptación al español. Procesamiento del lenguaje natural, 25-32.

Hsu, L. y Chen Y. J. (2020). Neuromarketing, subliminal advertising, and hotel selection: An EEG study. Australasian Marketing Journal, 200-208. https://doi.org/10.1016/j.ausmj.2020.04.009.

James, W. (1985). ¿What is an emotion? Studies in Psychology, (6)21, 57-73. https://doi.org/10.1080/02109395.1985.10821418.

Kleinginna, J. K y Kleinginna, N. (1981). A categorized list of emotion definitions, with suggestions for a consensual definition. Motivation and Emotion, 345-379.

Kumar, S., Yadava, M. y Roy, P. (2019). Fusion of EEG response and sentiment analysis of products review to predict customer satisfaction. Information Fusion, 52, 41-52. https://doi.org/10.1016/j.inffus.2018.11.001 
Núñez- Cansado, M., López López, A. y Vela Delfa, C.

Revisión teórico-cientifícia del marco conceptual de la emoción y el sentimiento y su aplicación al neuromarketing

Kumar, S. Y. (2019). Fusion of EEG response and sentiment analysis of products review to predict customer satisfaction. Information Fusion, 52, 41-52. https://doi.org/10.1016/j.inffus.2018.11.001

LeDoux, J. (1996). The emotional brain: The mysterious underpinnings of emotional life. Simon \& Schuster.

LeDoux, J. (2002). Synaptic Self: How Our Brains Become. Viking Books.

Lei Z., Yang Y. y Yang M. (2018). SAAN: una red de atención consciente del sentimiento para análisis de los sentimientos. SIGIR'18. 1197-1200. Arbor, USA. https://doi.org/10.1145/3209978.3210128.

Lewinski, P., Fransen, M. L. y Tan E. S. H. (2014). Predicting advertising effectiveness by facial expressions in response to amusing persuasive stimuli. ournal of Neuroscience, Psychology, E Economics, 7 (1), 1-14 .

Lim, M. (2018). Demystifying neuromarketing. Journal of Business Research, 105-220.

Lindquist, K. A., Wager, T. D., Kober, H., Bliss-Moreau, E. y Barrett, L. F. (2012). The brain basis of emotion: A meta-analytic review. Behavioural \& Brain Sciencies, 35 (3), 121-143.

Magnone, P. (2012). La alegoría del carro del alma en Platón y en la Kaṭha Upanișad. Exégesis y hermenéutica de obras tardoantiguas y medievales, 87-127.

Mahamud, K. (2018). Emociones y sentimientos: coordenadas históricas y multidicisplinares de un campo de estudios claves para la ciencia de la educación. Avances en supervisión educativa. Revistas de la asociación de inspectores de España., 118.

Mandler, G. (1975). The search of emotion. En L. Levi, Emotions: Their Parameters and Measurement. (121-148). Raven Press.

Marañón, G. a. (1920). La Emoción. Voluntad, vol. IX.

Marañón, G. b. (1920). La reacción emotiva a la adrenalina. La Medicina Íbera.

Martínez Herrador, J. L., Núñez-Cansado M. y Vadunquillo M. I. (2020). Metodología de neuromarketing: medición de Sociograph aplicada al análisis de la narrativa audiovisual erótica y sus aplicaciones a la estrategia de mercadotecnia. Vivat Academia, (150), 131-153. https://doi.org/10.15178/va.2020.150.131-153.

Melamed, A. (2016). Las teorías de las emociones y su relación con la cognición: un análisis desde la filosofía de la mente. Cuadernos de la facultad de humanidades y Ciencias Sociales. 49, 3-38. 
Núñez- Cansado, M., López López, A. y Vela Delfa, C.

Revisión teórico-cientifícia del marco conceptual de la emoción y el sentimiento y su aplicación al neuromarketing

Morín, C. (2011). Neuromarketing: The New Science of Consumer Behavior. Society, 131-135.

Neethu, R. y Rajasree, M. S. (2013). The 4th international conference on computing, communications and networking technologies. Sentiment analysis in twitter using machine learning techniques, 1-5. Tiruchengode. https://doi.org/10.1109/ ICCCNT.2013.6726818

Núñez-Cansado, M., López López, A., \& Caldevilla Domínguez, D. (2020). Situation of Neuromarketing Consulting in Spain. Frontiers in Psychology, 11, 1854. https://doi.org/10.3389/fpsyg.2020.01854

Olofsson J. K., Nordin S., Sequeira H. y Polich J. (2008). Affective picture processing: An integrative review of ERP findings. Biological Psychology, 247-265.

Thorson E. y Heide M. P. (1990). The memory impact of commercials varying in emotional appeal and product involvement. Emotion in Advertising. Quorum Books.

Pang L. y Lee B. (2004). A Sentimental Education: Sentiment Analysis Using Subjectivity Summarization Based on Minimum Cuts. Proceedings of the 42nd ACL. 271--278.

Papez, J. (1995). A proposed mechanism of emotion. 1937. Journal Neuropsychiatry Clinic, 7(1), 103-12. https://doi.org/10.1176/jnp.7.1.103

Plassmann, H., O'Doherty, J., Shiv, B. y Rangel, A. (2008). Marketing actions can modulate neural representations of experienced pleasantness. National Institutes of Health, 1050-1054. https://doi.org/10.1073/pnas.0706929105.

Plutchik, R., \& Ax, A. F. (1967). A critique of determinants of emotional state by schachter and singer (1962). Psychophysiology, 4(1), 7982. https://doi.org/10.1111/j.1469-8986.1967.tb02740.x

Poels, K. y Dewitte, S. (2006). How to capture the heart? Reviewing 20 years of emotion measurement in advertising. journal advertising research, 46, 18-37.

Rampl, L. V., Opitz, I. M., Welpe, I. M. y Kenning, P. (2016). The role of emotions in decision-making on employer brands: insights from functional magnetic. Springer Science+Business Media, 361-374.

Romero Moreno, F., Sanchez Martelo, C., Corredor B. Sánchez Cifuentes, F. y Ospina López, J. (2020). Análisis de sentimiento a las opiniones generadas. Risti, 187-203. 
Núñez- Cansado, M., López López, A. y Vela Delfa, C.

Revisión teórico-cientifícia del marco conceptual de la emoción y el sentimiento y su aplicación al neuromarketing

Ruanguttamanun, C. (2014). Neuromarketing: I put myself into a fMRI scanner and realized that I love Louis Vuitton ads. Procedia Social and Behavioral Sciencie, 211218. https://doi.org/10.1016/j.sbspro.2014.07.036.

Russell, J. (1980). A circumplex model of affect. Journal Personal Sociology Psychology, 1161-1178.

Salah, A., Sebe, N. y Gevers, T. (2009). Communication and automatic interpretation of affect from facial expressions. En D. \&. Gokay, Affective Computing and Interaction: Psychological, Cognitive and Neuroscientific perspectives 157-183. Hershey (USA): IGI Global.

Scarantino, A. (2018). Are LeDoux's suvirval circuits basic emotions under a diferente name? Behavioral Sciencies, 24, 75-82. https://doi.org/10.1016/j.cobeha.2018.06.001001

Scheutz, M. (2000). Surviving in a hostile multi-agent environment: how simple affective states can aid in the competition for resources. Proceedings of the Thirteenth Canadian Conference on Artificial Intelligence. 389-399. Montreal. Canada. https:// doi.org/10.1007/3-540-45486-133

Shapiro, A., Sudhof, M. y Wilson, D. (2020). Measuring news sentiment. Journal of Econometrics. https:// doi.org/10.3145/epi.2014.mar.07

Sherer, K. (1884). Aproaches of emotion. Psychology press. Taylor \& Francis Group.

Souza Baelar, L. (2011). Estudio de las emociones: una perspectiva transversal. Contribuciones a las Ciencias Sociales, 16-32.

Sun Y., Wang Z., Zhang B., Zhao W., Xu f., Liu J. y Wang B. (2020). Residents sentiments towards electricity price policy: Evidence from text mining in social media. Conservation and Recycling, (160). 104903. https://doi.org/10.1016/j.resconrec.2020.104903.

Trueba, C. (2009). La Teoría aristotélica de las emociones. Signos filosóficos, 1665-1324.

Vargas, A. y Espinoza, A. (2008). Pasión y Razón en Thomas Hobbes. Alpha. 26, 135152. http://dx.doi.org/10.4067/S0718-22012008000100009.

Vecchiato, G., Cherubino, P., Maglione, A. G., Ezquierro, M. T. H., Marinozzi, F., Bini, F., Trettel, A. y Babiloni, F. (2014). How to measure cerebral correlates of emotions in marketing relevant tasks. Cognitive Computation, 6(4), 856-871. https:// doi.org/10.1007/s12559-014-9304-x.

Vigotsky, L. (2004). Teoría de las emociones: Estudio histórico- Psicológico. Akal Universitaria. 
Núñez- Cansado, M., López López, A. y Vela Delfa, C.

Revisión teórico-cientifícia del marco conceptual de la emoción y el sentimiento y su aplicación al neuromarketing

Wahab, W., Ridwan, M. y Kusumoputro, B. (2015). Design and implementation of an automatic face-image data acquisition system using IP based multi camera. $\begin{array}{lll}\text { International Journal 1042-1049. } & \text { Techonology, }\end{array}$ https://doi.org/10.14716/ijtech.v6i6.1848.

Wilson, T. W. J. (2005). Proceedings of Human Language Technology Conference and Conference on Empirical Methods in Natural Language. Recognizing contextual polarity in phrase-level sentiment analysis. 347-354.

Yao, F. y Wang, Y. (2020). Domain-specific sentiment analysis for tweets during hurricanes (DSSA-H): A domain-adversarial neural-network-based approach. Computers, Environment and Urban Systems, 101522. https://doi.org/10.1016/j.compenvurbsys.2020.101522

Zurawicki, L. (2010). Neuromarketing: Exploring the brain of the consumer. Heidelberg: Springer.

\section{AUTOR/ES:}

\section{Marian Núñez-Cansado.}

Doctora en Ciencias de la Información, programa Ciencias de la Comunicación y Sociología. Licenciada en Ciencias de la información, especialidad Publicidad y Relaciones Públicas. Licenciada en Psicología, y Máster en Neuropsicología clínica. En la actualidad, es profesora Contratada Doctora en La Facultad de Ciencias Sociales Jurídicas y de la Comunicación en la Universidad de Valladolid.

Orcid ID: https:/ / orcid.org/0000-0002-6658-3996

\section{Aurora López López}

Doctora en Ciencias de la Información. Licenciada en Publicidad y Relaciones Públicas. En la actualidad profesora Doctora en La Facultad de Ciencias Sociales Jurídicas y de la Comunicación en la Universidad de Valladolid.

Orcid ID: https://orcid.org/0000-0001-8584-850X

\section{Cristina Vela Delfa}

Cristina Vela Delfa es Doctora en Ciencias del Lenguaje por la Universidad Complutense de Madrid y licenciada en Lingüística por la Universidad Complutense de Madrid. Es profesora Contratada Doctora en el Departamento de Lengua Española de la Universidad de Valladolid, en la Facultad de Ciencias Sociales Jurídicas y de la Comunicación.

Orcid ID: https:/ / orcid.org/0000-0002-4915-5260 